\title{
Corela
}

Cognition, représentation, langage

13-1 | 2015

Vol. $13, \mathrm{n}^{\circ} 1$

\section{Vers une analyse de l'apprentissage des langues en environnements virtuels immersifs}

\section{Hani Qotb}

\section{(2) OpenEdition}

\section{Journals}

Édition électronique

URL : http://journals.openedition.org/corela/3865

DOI : $10.4000 /$ corela.3865

ISSN : 1638-573X

\section{Éditeur}

Cercle linguistique du Centre et de I'Ouest - CerLICO

\section{Référence électronique}

Hani Qotb, « Vers une analyse de l'apprentissage des langues en environnements virtuels immersifs », Corela [En ligne], 13-1 | 2015, mis en ligne le 30 juin 2015, consulté le 19 avril 2019. URL : http:// journals.openedition.org/corela/3865; DOI : 10.4000/corela.3865

Ce document a été généré automatiquement le 19 avril 2019

\section{(c) (i) (2)(2)}

Corela - cognition, représentation, langage est mis à disposition selon les termes de la licence Creative Commons Attribution - Pas d'Utilisation Commerciale - Partage dans les Mêmes Conditions 4.0 International. 


\title{
Vers une analyse de l'apprentissage des langues en environnements virtuels immersifs
}

\author{
Hani Qotb
}

\section{1- Introduction}

1 Appelée aussi bain linguistique, l'immersion linguistique est une des approches didactiques qui permettent aux individus d'apprendre une langue étrangère tout en communiquant avec des natifs. Le Dictionnaire de didactique du français (2003) précise : "L'apprentissage d'une langue en immersion (bain linguistique) désignait un stage intensif au cours duquel toutes les activités de l'école et de la vie avaient lieu dans la langue cible. ". De leur côté, Cuq et Gruca (2003 : 309) soulignent le fondement didactique de l'immersion : «Le principe général de l'immersion est de tenter de récréer autant que possible les conditions d'appropriation naturelle d'une langue ". Autrement dit, l'immersion linguistique consiste à exposer l'apprenant longuement à la langue cible lors de situations de communication authentiques. Notons également que l'immersion linguistique peut avoir plusieurs modalités comme les séjours universitaire, linguistique et culturel. Dans un contexte mondialisé, la mobilité est désormais au centre de la politique linguistique de l'Union Européenne, dont l'objectif a été exprimé par le président de la commission européenne M. Barroso (2010) «Tout jeune devra avoir eu une opportunité de mobilité apprenante d'ici $2020 »$. Mais cette mobilité fait face à plusieurs difficultés (Halimi, S., et al., 2012) : réticence des parents à laisser partir leurs enfants, méconnaissance des dispositifs en vigueur, surcroit de travail, investissement fort de la part des enseignants, manque de motivation des apprenants et des enseignants, etc. Une telle situation pousse souvent certains apprenants à renoncer aux séjours linguistiques ou universitaires en se contentant de suivre des cours de langue dans leurs pays d'origine. 
2 Depuis la fin $\mathrm{du} 20^{\mathrm{e}}$, on assiste aux développements importants des Technologies de l'Information et de la Communication (TIC) qui ne cessent d'occuper le devant de la scène dans tous les domaines notamment dans les milieux éducatifs. Ces derniers s'intéressent de plus en plus aux différentes potentialités des TIC, surtout à celles d'Internet qui a réussi à favoriser la mobilité virtuelle et une culture nomade numérique (réseaux sociaux, blogs, espaces partagés de travail, etc.) pour un grand nombre d'apprenants quels que soient leur pays et leur âge. Grâce aux développements de la Réalité Virtuelle (Fuch, et al. , 2003), on constate la présence croissante des environnements virtuels 3D. Ceux-ci permettent de proposer un apprentissage immersif des langues qui est à la fois accessible, efficace et moins coûteux tout en favorisant les échanges entre des locuteurs natifs et les apprenants dans des situations de communication réelles. Cet article se propose de mener une analyse de l'apprentissage immersif de langue en environnement virtuel en vue de comprendre ses différentes dimensions. Dans un premier temps, nous présenterons certains exemples de formations immersives de langue en ligne. Ensuite, en se fondant sur le modèle SPEAKING élaboré par Hymes (1984), nous analyserons les composantes de l'apprentissage de langue en environnement virtuel immersif (EVI).

\section{2- Des projets d'apprentissage des langues en environnement virtuel immersif}

3 Lors de notre recherche sur la Toile, nous avons trouvé plusieurs exemples d'apprentissage des langues en environnements virtuels comme :

\section{2-1 l'Environnement Virtuel pour l'Enseignement Immersif 3D des langues étrangères (EVEIL 3D)'}

4 Financé par l'Union Européenne, ce projet est en cours d'expérimentation. Il concerne essentiellement la région Métropolitaine Trinational du Rhin Supérieur. Cet EVI a pour objectif de :

* développer une application pratique basée sur les outils de réalité virtuelle pour

l'apprentissage des langues étrangères ;

* sensibiliser les enseignants de langue à l'utilisation de 3D dans le cadre de

l'apprentissage scolaire ;

* promouvoir l'utilisation durable des résultats du projet.

Quant au monde virtuel du projet EVEIL, il se compose de trois dispositifs :

A- Une télévision 3D où l'image gagne en profondeur à tel point que certains objets

sortent de l'écran pour venir à la rencontre de l'utilisateur,

B- Un casque immersif facilitant la visualisation sans contraintes du moniteur,

C- Une projection de l'image sur un mur dans une Cave, une sorte de simulateur géant.

Les chercheurs de ce projet sont en train de mettre en place une formation immersive de langue en s'appuyant sur les hypothèses suivantes (Schlemminger, G. et al., 2012) :

H1 : Le degré d'immersion dans un environnement virtuel influence la compréhension d'énoncés oraux en langue étrangère.

H2 : Le degré d'immersion dans un environnement virtuel influence la mémorisation d'énoncés oraux en langue étrangère.

H3 : Le degré d'interaction dans un environnement virtuel influence la compréhension d'énoncés en langue étrangère. 
H4 : Le degré d'interaction dans un environnement virtuel influence la mémorisation d'énoncés oraux en langue étrangère.

6 Pour vérifier ces hypothèses, le groupe de recherche compte mener une expérience de formation de Français Langue Etrangère (FLE) auprès de 75 apprenants ayant de 13 à 14 ans dans un collège allemand. Adoptant une approche actionnelle, le scénario d'apprentissage propose aux apprenants un stage de vente dans lequel ces derniers effectuent des tâches particulières selon les consignes données par le tuteur (ex : ranger des articles sur les étagères du magasin virtuel).

\section{2-2 Avatar Languages}

7 Il s'agit d'un centre de langues qui présente des cours en ligne grâce à l'utilisation de plusieurs outils dont Second Life (SL). Dans une optique commerciale, ce centre vante les mérites de cet outil dans l'apprentissage des langues, notamment l'immersion, l'engagement et le contexte social, qui permettent de créer les conditions favorables pour réaliser les objectifs visés en langue cible. Créé par Howard Vickers, Avatar Languages adopte l'approche actionnelle en proposant aux apprenants certaines tâches favorisant l'interaction au sein de la communauté d'apprentissage. Appelée aussi " Quêtes sur Réelles ", cette approche combine l'enseignement des langues sur SL avec des activités de la vie réelle. Par exemple, on demande aux apprenants de réaliser une interview avec un spécialiste dans un domaine donné. Une telle activité journalistique amène l'apprenant à s'entrainer à parler, à écouter, à écrire et à lire en langue étrangère.

\section{2-3 Les Eonautes²}

8 Ce site a élaboré un jeu sérieux immersif visant à apprendre le FLE en ligne. Il adopte les niveaux de langue proposés par le CECR (2000) en amenant l'apprenant à développer essentiellement trois compétences communicatives (lire, écrire et écouter) à travers la participation à des activités variées. "Le déroulement de notre approche, précise-t-il , s'appuie sur les principes de la perspective actionnelle : l'apprenant devra mobiliser un certain nombre de savoirs et de savoir-faire pour accomplir les différentes tâches, réussir sa mission et ainsi franchir les étapes $d u$ jeu-apprentissage ${ }^{3} »$. Cet apprentissage a deux objectifs principaux à savoir : l'interaction et l'immersion. Pour participer à ce jeu sérieux, l'apprenant incarne un personnage imaginaire chargé de réaliser une tâche (une mission) à partir d'un scénario préalable. Les Eonautes proposent ainsi un support ludique où les participants sont introduits dans un univers graphique qui contextualise visuellement les missions à accomplir. Notons que les échanges se font uniquement par écrit grâce au chat disponible sur la plateforme. Le site propose plusieurs jeux sérieux en FLE comme Chez les Gaulois. Dans le cadre de trois voyages, l'apprenant sera amené à découvrir le marché et le système $d u$ troc, les recettes du village et travailler avec le poissonnier du marché. Le guide pédagogique détermine les objectifs communicatifs, grammaticaux et lexicaux de chaque séquence.

9 A partir de ces trois exemples mentionnés ci-dessus, nous tenterons d'analyser la situation dans laquelle se déroule l'apprentissage immersif de langue en mettant essentiellement l'accent sur ses composantes pour mieux cerner ses différentes dimensions. 


\section{3- Les composantes de l'apprentissage immersif de langue en environnement virtuel}

Dans le cadre de notre recherche sur l'apprentissage immersif des langues en ligne, il nous semble important d'analyser ses différents aspects en vue de mieux comprendre son fonctionnement, ses paramètres contextuels, les rôles de ses acteurs, etc. Apprendre une langue étrangère se fait grâce à un échange entre des partenaires (enseignant et apprenants) dans une situation de communication donnée. C'est pourquoi, nous avons recours au modèle de SPEAKING (Hymes D., 1984) ayant pour objectif d'analyser essentiellement toute situation de communication. Ce modèle introduit la notion de compétence de communication comme compétence complexe et qui dépasse largement la dimension linguistique en prenant en compte de certains éléments (le cadre, les participant, etc.) directement liés à la situation sociale dans laquelle l'interaction a lieu. La prise en compte de ces différents éléments permet de mieux comprendre le sens visé par les interlocuteurs impliqués dans les échanges. Le modèle de Hymes nous semble utile pour analyser les différentes dimensions de la situation de l'apprentissage en EVI. Ses huit composantes proposées facilitent la compréhension des interactions produites au sein de la communauté d'apprentissage en ligne. En appliquant ce modèle d'analyse sur la formation immersive des langues sur Internet, celle-ci pourrait comprendre les éléments suivants :

\section{3-1 Le cadre spatio-temporel " S : Settings "}

11 Le cadre spatio-temporel constitue une des composantes importantes dans laquelle se déroule l'apprentissage immersif des langues. Il s'agit d'un dispositif énonciatif qui permet aux interlocuteurs (apprenants et cadre enseignant) de co-produire leurs échanges dans les différentes situations. A propos de l'espace, on distingue deux environnements principaux : l'un est physique tandis que l'autre est virtuel. D'un côté, l'utilisateur (U) de l'EVI a une présence physique dans le monde réel. D'un autre côté, le monde virtuel regroupe les avatars (A) qui permettent aux utilisateurs d'interagir avec leurs pairs :

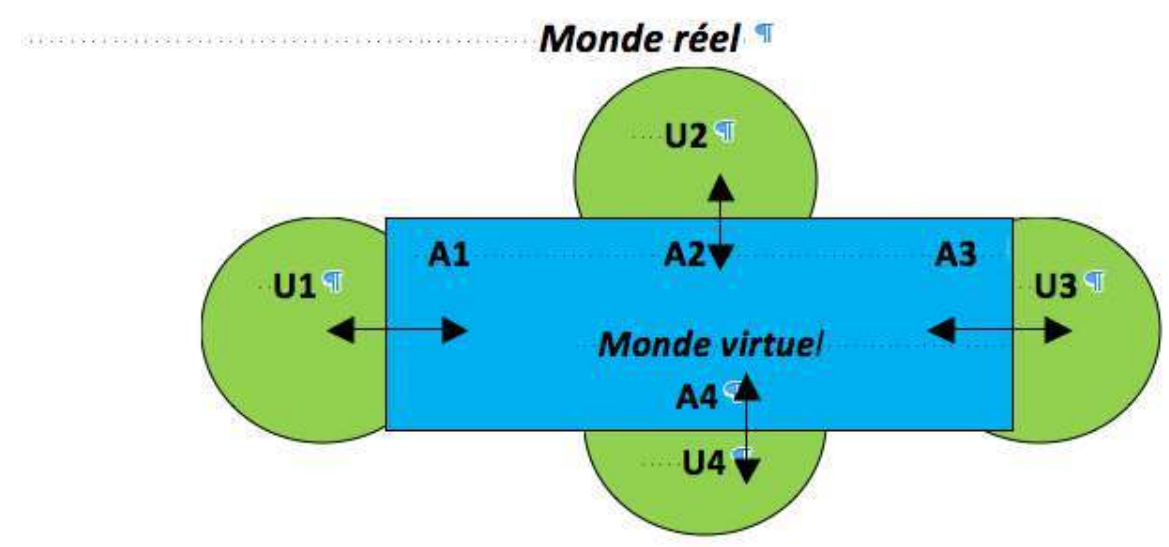

Figure 1: Les dimensions spatiales dans un apprentissage de langue en EVI

Dans ce contexte, il s'avère important de souligner les relations réciproques entre les actions produites dans le monde réel et celles du monde virtuel. L'utilisateur agit, via son 
avatar, dans les différentes situations à partir de ses propres connaissances, sa culture (occidentale, asiatique, etc.) et son caractère (agressif, amical, etc.). Dans l'autre sens, l'utilisateur est en mesure d'acquérir de nouvelles connaissances linguistiques et de développer les compétences communicatives dans un domaine donné. Précisons que l'accès à l'environnement peut être ouvert ou restreint selon la communauté d'apprentissage visée. Quant au temps dans l'apprentissage immersif en ligne, on distingue trois temporalités principales :

* Temporalité synchrone : les utilisateurs interagissent en temps réel (interactions orales et gestuelles)

$14{ }^{*}$ Temporalité quasi-synchrone : il s'agit des échanges via le clavardage (interactions écrites),

* Temporalité asynchrone : la communication se déroule en temps différé (interactions orales et écrites)

16 Notons que chaque temporalité est liée à un mode de communication particulier. Par exemple, les participants peuvent avoir recours à la temporalité synchrone, qui suppose des interactions orales et gestuelles en vue d'avoir des échanges directs lors de situations de communication. Ces trois temporalités rendent les interactions plus riches dans la mesure où chaque participant est capable de choisir la temporalité qui lui convient lors de différentes situations de communication. Ceci ne fait que donner une certaine " plasticité" aux échanges produits sans obliger les acteurs à utiliser une temporalité particulière.

\section{3-2 Les participants "P: participants"}

\begin{tabular}{|l|l|}
\hline $\begin{array}{l}\text { Fonction } \\
\text { pédagogique }\end{array}$ & $\begin{array}{l}\text { Faciliter l'apprentissage, éveiller aux concepts critiques. Encourager les } \\
\text { étudiants à argumenter et à construire leur savoir }\end{array}$ \\
\hline $\begin{array}{l}\text { Fonction } \\
\text { métacognitive }\end{array}$ & $\begin{array}{l}\text { Favoriser la réflexion métacognitive. Susciter des questionnements et } \\
\text { une analyse de leurs propres processus d'apprentissage. }\end{array}$ \\
\hline $\begin{array}{l}\text { Fonction } \\
\text { motivationnelle }\end{array}$ & $\begin{array}{l}\text { Encourager et motiver. Créer un environnement d'apprentissage amical } \\
\text { travailler pour la même cause }\end{array}$ \\
\hline $\begin{array}{l}\text { Fonction } \\
\text { organisationnelle }\end{array}$ & $\begin{array}{l}\text { Préparer un agenda, résumer et clarifier ce qui se dit, exprimer le } \\
\text { consensus qui se dégage ou proposer un vote formel }\end{array}$ \\
\hline Fonction évaluative & $\begin{array}{l}\text { Evaluer. Communiquer les objectifs et les critères d'évaluation. Rédiger } \\
\text { des commentaires dans une perspective d'évaluation formative }\end{array}$ \\
\hline Fonction technique & $\begin{array}{l}\text { Faire en sorte que les étudiants soient à l'aise avec le système technique, } \\
\text { le but ultime étant de rendre l'appareillage technique transparent }\end{array}$ \\
\hline
\end{tabular}

Tableau 1: Les six fonctions du tuteur (Decamps S., De Lièvre B. et Depover C., 2006)

17 A ces fonctions s'ajoutent deux autres fonctions spécifiques au tuteur de langue. La première est linguistique dans la mesure où le tuteur se charge de développer les compétences communicatives (orale et écrite), d'expliquer les termes difficiles, de 
corriger les productions orales et écrites, de faciliter la compréhension de certaines règles grammaticales, etc. Quant à la deuxième fonction, elle est didactique puisque le tuteur explique aux apprenants les objectifs à atteindre, l'approche pédagogique adoptée, le rôle de chaque acteur, les activités à réaliser, etc. Notons également que l'EVI donne la possibilité aux apprenants d'entrer en contact avec des natifs qui ne font pas partie de la formation comme c'est le cas de Second Life. Une telle possibilité facilite l'accès à des situations de communication authentiques.

\section{3-3 Les objectifs "E: Ends"}

18 Fixer des objectifs est une étape fondamentale lors de l'élaboration d'un cours de langue dans un apprentissage immersif en ligne. Ces objectifs sont essentiellement liés à l'intention de chaque participant à la formation. Dans ce contexte, on distingue deux types d'intention. D'un côté, l'intention des apprenants qui cherchent à développer certaines compétences linguistiques et communicatives en langue cible. C'est pourquoi, il est primordial de donner la possibilité aux apprenants d'exprimer leurs besoins avant le début de la formation grâce à des grilles d'analyse et des entretiens. D'un autre côté, le cadre enseignant (formateur-tuteur) est marqué par l'intention de satisfaire les besoins de ses apprenants tout en les aidant à atteindre leurs objectifs. La rencontre de ces deux intentions donne naissance à la formation immersive de langue en ligne. Précisons que les objectifs des participants ne sont pas figés mais qu'ils peuvent changer selon les développements du cours suivi, d'où la nécessité de répondre aux nouveaux besoins qui pourraient émerger lors de la formation.

\section{3-4 Les actes du langage "A : Acts"}

Il s'agit des interactions produites entre les participants dans les différentes situations de communication. L'apprenant de langue est amené à réaliser des échanges avec son formateur et ses pairs dans le but de développer ses compétences et ses connaissances en langue cible. Rappelons que l'idée de l'immersion linguistique repose essentiellement sur la volonté de placer l'apprenant dans « un bain linguistique » pour qu'il puisse favoriser les situations de communication. Les actes du langage peuvent avoir plusieurs formes en allant d'un simple énoncé à la simulation globale en passant par des dialogues, des réunions de travail, des conversations informelles, etc. Dans un cours de langue en ligne, l'acte du langage a deux acceptions principales. D'une part, le langage est le moyen principal qui permet aux participants d'interagir dans les différentes situations (Herring, S.-C., 2004). D'autre part, il constitue le produit recherché dans une formation de langue dans la mesure où les toutes les activités proposées devraient permettre à l'apprenant de réaliser des actes du langage acceptables aux niveaux lexical, grammatical et sémantique tout en prenant en compte les paramètres contextuels (statut, âge, but, cadre spatiotemporel, etc.).

Le formateur peut accorder une importance particulière à certains actes du langage selon les besoins de ses apprenants et les objectifs de la formation suivie. En EVI, on assiste à interactions multimodales qui sont le fruit de l'utilisation de plusieurs canaux de communication : l'oral, l'écrit et le gestuel. Ces canaux sont complémentaires, ce qui rend les interactions plus proches de la réalité, augmentant ainsi le sentiment d'immersion dans l'apprentissage de langue. Ajoutons aussi que l'acte de langage en EVI participe d'un 
discours polylogue dans la mesure où plusieurs interlocuteurs participent à sa production. Il peut être aussi un dialogue lors de la présence de deux interlocuteurs à la situation de communication. Les deux types de discours renforcent ainsi la notion de la communauté qui est fondamentale dans l'apprentissage de langue en EVI.

\section{3-5 La tonalité "K : Keys"}

21 La tonalité concerne les aspects psychologiques de l'échange entre les différentes partenaires dans une situation de communication donnée. Cet échange peut être tendu, agressif, amical, professionnel, intime, etc. selon la nature des relations entre les interlocuteurs. Par exemple, l'apprenant a tendance à avoir des échanges détendus et amicaux avec ses pairs et d'autres plus formels avec son enseignant. Dans ce contexte, il s'avère nécessaire de créer une ambiance conviviale pour renforcer les liens socioaffectifs au sein de la communauté d'apprentissage afin que les apprenants soient à la fois motivés et impliqués au cours de différentes étapes de la formation suivie.

\section{3-6 Les instruments de communication en ligne "I : Instrumentalities"}

Il s'agit des différents moyens de communication mis en place pour faciliter les interactions entre les interlocuteurs. Dans un apprentissage immersif de langue en ligne, les participants ont recours, chacun selon ses besoins et le contexte de l'échange, à plusieurs formes de communication : audio, écrite et gestuelle. L'utilisation de ces formes de communication renforce le sentiment d'immersion chez les apprenants. De son côté, le formateur peut privilégier une forme de communication particulière pour améliorer les compétences communicatives visées. Par exemple, pour développer la compétence orale chez les apprenants, il s'intéresse à mettre en place certaines activités comme des dialogues, des jeux de rôle, des simulations, etc. Il est nécessaire que les participants passent un certain temps à se familiariser à l'utilisation de différents canaux de communication pour mieux gérer leurs interactions dans les situations de formation immersive.

\section{3-7 Les normes " $\mathrm{N}$ : norms"}

Dans une formation immersive de langue en ligne, les normes concernent les règles de comportement linguistique et social comme les rituels suivis dans les situations de communication. En fait, les normes permettent de créer un cadre commun dans lequel les apprenants et le cadre enseignant participent aux différentes activités. Ce cadre commun a trois dimensions principales. La première est linguistique dans la mesure où les participants sont amenés à respecter les règles lexicales, grammaticales, etc. pour pouvoir communiquer avec les autres en langue-cible. La deuxième dimension est sociale, elle porte sur les rites relationnels à prendre en compte au cours des échanges : âge, sexe, statut social et professionnel, etc. Par exemple, l'apprenant a tendance à utiliser le vouvoiement avec le cadre enseignant alors qu'il tutoie ses pairs. La troisième dimension concerne les règles liées à l'environnement technique et juridique d'Internet. Lors d'une simulation en EVI, les utilisateurs doivent respecter un code de «bonne conduite » et de " savoir communiquer » (Marcoccia, 2000 : 252). Appelée aussi la nétiquette, ce code de bonne conduite concerne "l'ensemble des conventions de bienséance régissant le comportement des internautes dans le réseau " (Idem). Le même auteur met en évidence certaines 
recommandations à prendre en compte lors des interactions en ligne : les principes de politesse, le maxime de clarté et les règles liées à l'environnement technique et juridique d'Internet.

\section{3-8 Les genres "G : Genres"} linguistiques et communicatives visées. Appelés genres, ces types de discours prennent en considération des conventions langagières et sociales préalables. C'est le cas par exemple de courriers administratifs, recettes de cuisine, publicité, entretiens, etc. Dans un cours de Français sur Objectifs Spécifiques (FOS), le formateur peut proposer à des apprenants de réaliser une simulation d'entreprise où ils peuvent avoir deux types de discours. L'un est formel. Il s'agit des échanges entre le directeur et ses employés sur les activités commerciales de l'entreprise. L'autre est informel portant sur les interactions entre les employés sur des thèmes de la vie quotidienne.

\section{4- Conclusion}

La prise en compte des composantes mentionnées ci-dessus facilite la compréhension du déroulement de l'apprentissage immersif des langues en environnement numérique. Cette analyse permet aux chercheurs de mieux déterminer les différentes dimensions de la formation immersive en lige: le cadre spatio-temporel, les rôles de différents acteurs, les objectifs à atteindre, etc. Elle peut aussi avoir des retombées positives sur les participants à la formation immersive. D'une part, elle facilite au cadre enseignant la scénarisation des cours de langue en EVI dans le but d'atteindre les objectifs visés. D'autre part, elle favorise la motivation et la participation des apprenants puisque qu'ils sont conscients du contexte de formation, des outils de communication utilisés, des normes linguistiques et sociales à respecter, etc. Notons que l'apprentissage de langue en EVI peut se faire dans deux cadres : l'un est formel (autonomie limitée ou guidée) tandis que l'autre est informel (autonomie totale). Dans les deux cas, ce type d'apprentissage est devenu une des entrées pour communiquer en langue étrangère dans un contexte marqué par la montée en puissance des réseaux sociaux. C'est pourquoi, les EVI ne font que favoriser la mobilité virtuelle d'autant plus que ces réseaux sont interconnectés, amenant ainsi l'apprenant à faire face à des situations de communication authentiques pour une meilleure pratique de la langue-cible.

\section{BIBLIOGRAPHIE}

BARRoSo, J. M. (2010) Lancement d'une nouvelle Stratégie pour 2020, Bruxelles, 3 mars, in HALIMI, S. (dir.) (2012) Apprendre les langues, apprendre le monde, rapport présenté au ministre de l'Education nationale, disponible sur : http://media.education.gouv.fr/file/02_Fevrier/91/5/Apprendre-leslangues-Apprendre-le-monde_206915.pdf

Corela, 13-1 | 2015 
Cadre Européen Commun de Références (2000), Division des politiques linguistiques, Bruxelles. CELIK, C. (2007) «Les modalités du tutorat dans un campus numérique de maîtrise de langue française entièrement à distance ». Actes du Colloque d'EPAL, Grenoble, 7-8-9 juin. Disponible sur : http://w3.u-grenoble3.fr/epal

CUMMINS, J., \& SWAIN, M. (1986) Bilingualism in education : aspects of theory, research and practice. London : Longman

CUQ, J.-P. (dir.) (2002) Le Dictionnaire de didactique du français, Clé Internationale.

CUQ, J.-P. \& GRUCA, I. (2003) Cours de didactique du français langue étrangère et seconde, PUG.

DAELE, A. \& DOCQ, F. (2002) « Le tuteur en ligne, quelles conditions d'efficacité dans un dispositif d'apprentissage collaboratif à distance ? ", 19ème colloque de l'AIPU, Louvain-la-Neuve, mai. Disponible sur : http://www.ipm.ucl.ac.be/articlesetsupportsIPM/DocqDaeleAIPU2002.pdf DECAMPS, S., De LIÈVRE, B. et DEPOVER, C. (2006) « Les tuteurs tels qu'ils se définissent dans l'exercice de leurs fonctions » CEMAFORAD 3, Sousse. Disponible sur : http://ute.umh.ac.be/ site_ute2/menu.htm

GODINET, H. \& CARON, C. (2003) «L'accompagnement du processus d'apprentissage dans le Campus Numérique FORSE: modalités et outils. ", Actes de la Conférence d'Environnements Informatiques pour l'Apprentissage Humain, Strasbourg, EIAH Atief-INRP, pp.223-234.

FUCHS P. \& ARNALDI B.b (2003) «Tisseau J. La réalité virtuelle et ses applications » In Fuchs P. (éd.) \& Moreau G. (coord.) Le traité de la réalité virtuelle. Volume 1 : fondements et interfaces comportementales. Presse de l'Ecole des Mines de Paris, pp. 3- 52.

HAGÈGE, C. (1996) L'enfant aux deux langues, Paris, Editions Odile Jacob.

HALIMI, S. (dir.) (2012) Apprendre les langues, apprendre le monde, rapport présenté au ministre de l'Education nationale, disponible sur : http://media.education.gouv.fr/file/02_Fevrier/91/5/ Apprendre-les-langues-Apprendre-le-monde_206915.pdf HERRING, S.-C. (2004) «Computer-Mediated Discourse Analysis: An Approach to Researching Online Behavior». In Barab, S.-A, Kling, R. \& Gray, J.-H (éds.), Designing for Virtual Communities in the Service of Learning, pp.338-376. New York : Cambridge University Press.

HYMES, D., (1984) Vers la compétence de communication, Paris, Didier.

MARCoccia, M. (2000) «Les Smileys : une représentation iconique des émotions dans la communication médiatisée par ordinateur ». In Plantin, C., M. Doury, Traverso, V. (éd.), Les émotions dans les interactions communicatives. Lyon : ARCI - Presses Universitaires de Lyon, pp.249-263.

PORCHER, L. (1994) « L'enseignement de la civilisation », Revue Française de Pédagogie, N¹08, JuilletAoût-septembre, 5-12.

PUREN, C. (2002) « Perspectives actionnelles et perspectives culturelles en didactique des languescultures : vers une perspective co-actionnelle co-culturelle ", $n^{\circ} 3 / 2002$ des Langues modernes, juil.août-sept. pp. 55-71, Paris, APLV.

QОТВ, Н. (2012) La scénarisation des cours de langue en ligne : spécificités, démarches, perspectives, Edition Presses Universitaires Européennes, Saarbrücken. 
SCHLEMMinger, G. et al., (2012) « Réalité virtuelle et éducation : l'exemple de l'apprentissage des

langues », Disponible sur : http://www.eveil-3d.eu/

downloads/2012-10-29__Journees_realite_virutelle_LSIIT(1).pdf

Van DIXHOORN, L., LOISEAU, M., MANGENOT, F., POTOLIA, A. et zourou, K. (2010) Apprentissage des

langues: ressources et réseaux. Etude du consortium Language learning and social: 6 key dialogues.

Disponible sur : http://www.elearningeuropa.info/files/LS6/aprentissage\%20des\%20langues\%

20ressources\%20et\%20reseaux\%20DEF.pdf

http://www.v-lang.eu/section/home/

http://www.avatarlanguages.com/fr/index.php http://www.eonautes.com/fr/edu/index.html http://www.eveil-3d.eu/francais/93.php

\section{NOTES}

1. http://www.eveil-3d.eu/francais/93.php, consulté le 25/9/2015

2. http://www.eonautes.com/fr/edu/index.html, consulté le 25/5/2015

3. http://www.eonautes.com/fr/edu/index.html, consulté le 25/5/2015

\section{RÉSUMÉS}

L'immersion linguistique est une des approches didactiques qui permettent aux individus d'apprendre une langue étrangère. Grâce aux développements des Technologies de l'Information et de la Communication (TIC), on constate la présence croissante des Environnements Virtuels Immersifs (EVI) qui donnent la possibilité de proposer des cours de langue en ligne. En adoptant le modèle SPEAKING de Hymes (1984), nous tentons d'analyser les différentes composantes des formations immersives des langues sur Internet en mettant l'accent sur les éléments suivants : le cadre spatio-temporel, les participants, les objectifs, les actes du langage, les tonalités, les instruments, les normes et les genres. La prise en compte de ces éléments facilite la compréhension du déroulement de l'apprentissage immersif sur Internet tout en aidant le cadre enseignant à mieux scénariser des cours de langue en EVI dans le but d'atteindre les objectifs visés.

Language immersion is one of the didactic approaches that enable individuals to learn a foreign language. Owing to development in Information and Communication Technologies (ICT), Immersive Virtual Environments (IVE) have emerged, and these make online language courses possible. With Hymes' (1984) SPEAKING model, we analyse the different components of immersive Internet language training focusing on the following points: settings, participants, aims, language acts, tonality, instrumentality, norms and genders. Taking into account these elements facilitates the understanding of immersive Internet learning while helping instructors improve scenarising language courses within IVE so as to achieve learning goals 
INDEX

Mots-clés : Apprentissage des langues, Immersion linguistique, TIC, Environnement Virtuel Immersif, Modèle de SPEAKING

Keywords : Language learning, language immersion, ICT, Immersive Virtual Environments, SPEAKING model

\section{AUTEUR}

\section{HANI QOTB}

Paxiling 5267 CNRS / Montpellier III

Hani.qotb@univ-montp.3.fr 